\title{
Pseudothermoosmosis in a Composite Membrane System
}

\author{
L. D'Ilario and M. Canella \\ Laboratori Ricerche di Base, Snamprogetti S.p.A., \\ 00015 Monterotondo, Rome, Italy.
}

(Received October 7, 1976)

\begin{abstract}
A composite membrane system, constituted by two cellulose membranes and an aqueous solution of poly(ethylene oxide) (liquid membrane), was used to investigate the transport phenomena occurring when a thermal difference was applied. At the steady state, pressures were measured $\left(c a .6 .0 \times 10^{5} \mathrm{dyn}^{-2}\right)$ far higher than those reached in ordinary thermoosmosis experiments. In order to explain these unusual effects a model was proposed in which the contribution of the polymeric liquid membrane was taken into account. The thermodynamics of irreversible processes was used to derive the composite membrane phenomenological equations containing both the classical thermoosmosis and the thermal diffusion as particular cases. In this paper the observed phenomena were indicated as "pseudothermoosmosis." The flow of matter, phenomenological coefficients and heat of transport were also measured. For the activation energy a value of $3.91 \mathrm{kcal} \mathrm{mol}^{-1}$ was found, indicating the diffusive nature of the solvent flow through the composite membrane.

KEY WORDS Membranes / Thermoosmosis / Transport Phenomena / Thermodynamics of Irreversible Processes /
\end{abstract}

Transport phenomena occurring across membranes separating two solutions of identical composition, kept at two different temperatures, have already been studied, and they are known as thermoosmotic effects. ${ }^{1-4}$

The temperature difference $(\Delta T)$ between the two semicelles of constant volume separated by a membrane, causes a flow of matter $J_{M}$ which is called "thermoosmotic flow." At the steadystate a pressure difference $(\Delta p)$, called "thermoosmotic pressure," is established. Coupled to the flow of matter $J_{\mathrm{M}}$ is a heat flow $J_{q}$. The thermodynamics of irreversible processes can be used to treat such a nonisothermal system by the phenomenological equations ${ }^{1-4}$

$$
\begin{gathered}
J_{\mathrm{M}}=-L_{11} \frac{v}{T_{\mathrm{m}}} \Delta p-L_{12} \frac{1}{T_{\mathrm{m}}{ }^{2}} \Delta T \\
J_{q}=-L_{21} \frac{v}{T_{\mathrm{m}}} \Delta p-L_{22} \frac{1}{T_{\mathrm{m}}{ }^{2}} \Delta T
\end{gathered}
$$

where $v$ is the specific volume of the permeant, $T_{\mathrm{m}}$ is the mean temperature, $L_{11}$ is related to the permeation through the membrane, and is called "permeability constant," $L_{22}$ is the "thermal conductivity," $L_{12}$ and $L_{21}$ are crosscoefficients for which the Onsager reciprocal relation applies.

$$
L_{i k}=L_{k i}
$$

The ratio $L_{12} / L_{11}$, called "heat of transport," is indicated by $Q^{*}$ :

$$
Q^{*}=\frac{L_{12}}{L_{11}}=-v T_{\mathrm{m}}\left(\frac{\Delta P}{\Delta T}\right)_{J_{\mathrm{M}}=0}
$$

The equations stated above are valid for systems in which the membrane is thin enough and behaves inertly, just like a separating wall with a certain permeability to the solvent molecules.

The chemical potential difference in the equation defining the driving force for the flow of matter will be in this case reduced to

$$
\Delta \mu=v \Delta p-S \Delta T
$$

in which $S$ is the specific entropy.

Experiments carried out by several research$\mathrm{ers}^{5-12}$ show that the amplitude of the thermo- 
osmotic effect is very small, being in the range of a few centimeters of water. By replacing the single cellulose membrane with a composite membrane system behaving not inertly under the temperature gradient imposed, we could observe a 60 -fold amplification of the thermoosmotic effect. ${ }^{13}$

We define as composite membrane a system in which a solution of macromolecule is kept between two cellulose membranes permeable to the solvent only.

The aim of the present paper is to verify if the phenomenological eq 1 and 2 describing the classical thermoosmosis could be applied to the composite membrane system.

\section{EXPERIMENTAL}

\section{Materials}

Poly(ethylene oxide). The poly(ethylene oxide) (PEO) used in our experiments was purchased from Fluka A.G. The average molecular weight was 20,000 .

\section{Membranes}

We have used dialysis membranes A.H. Thomas Co. $\mathrm{N}^{\circ} 4465-\mathrm{A} 2$ of pure cellulose, with approximately $0.1 \%$ of sulfur. The pore diameter was $48 \AA$, and the thickness $100 \mu \mathrm{m}$. The molecular weight cut off was 12,000 .

\section{Apparatus}

A drawing of the experimental apparatus may be seen in Figure 1. It consisted of

-a plexiglass cylindrical chamber (LM) 4-mm thick, with the inner diameter of $30 \mathrm{~mm}$, filled with a PEO aqueous solution;

-two cellulose membranes $\left(\mathrm{m}_{1}, \mathrm{~m}_{2}\right)$ on both sides of LM;

-two solvent reservoirs $\left(R_{1}, R_{2}\right)$ equipped with heating jackets $\left(h_{1}, h_{2}\right)$ connected to two thermostats. The reservoir $R_{2}$ was kept at the lower temperature in order to minimize convective flows;

- a movable solvent reservoir $R_{3}$, connected to $R_{2}$ by means of a tube with a diameter of $3 \mathrm{~mm}$, to keep constant the hydrostatic pressure on the lower side of $\mathrm{m}_{2}$.

To avoid leakages, rubber rings were used between the reservoirs and the membranes. The apparatus allowed no leakage up to about 20 atmospheres.

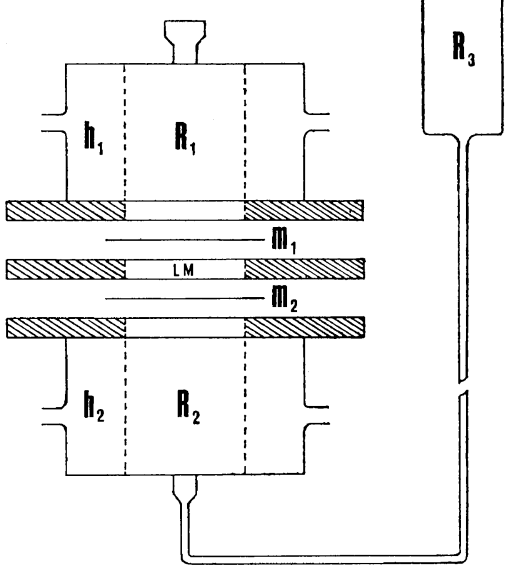

Figure 1. Experimental apparatus: $\mathbf{R}_{1}, \mathbf{R}_{2}, \mathbf{R}_{3}$, solvent reservoirs; $h_{1}, h_{2}$, heating jackets; $m_{1}, m_{2}$, cellulose membranes; LM, plexiglass chamber containing the polymer solution.

\section{Pressure Measurements}

Pressure transducers, supplied by Bell \& Howell Ltd. and mercury capillary manometers were used for pressure measurements. The instrumental error was in any case lower than $\pm 1 \mathrm{mmHg}$.

\section{Flow Measurements}

The solvent flow was measured by means of a horizontal capillary $50-\mathrm{cm}$ long with an internal diameter of $1 \mathrm{~mm}$. In order to minimize the solvent evaporation, cold water was allowed to circulate inside a transparent mantle winding the capillary.

\section{Temperature Difference Measurements}

The difference of temperature on the two sides of the composite membrane was determined by means of calibration curves obtained by measuring $T_{1}$ and $T_{2}$ on a test cell and varying the thermostats temperatures. The temperature measurements were carried out with copperconstantan thermocouples placed on the two membranes $m_{1}$ and $m_{2}$. The thermostats stability was within $\pm 0.05^{\circ} \mathrm{C}$. The experimental error to be attributed to the temperature difference measurements is $\pm 0.5^{\circ} \mathrm{C}$.

\section{RESULTS AND DISCUSSION}

All our experiments were carried out at a 


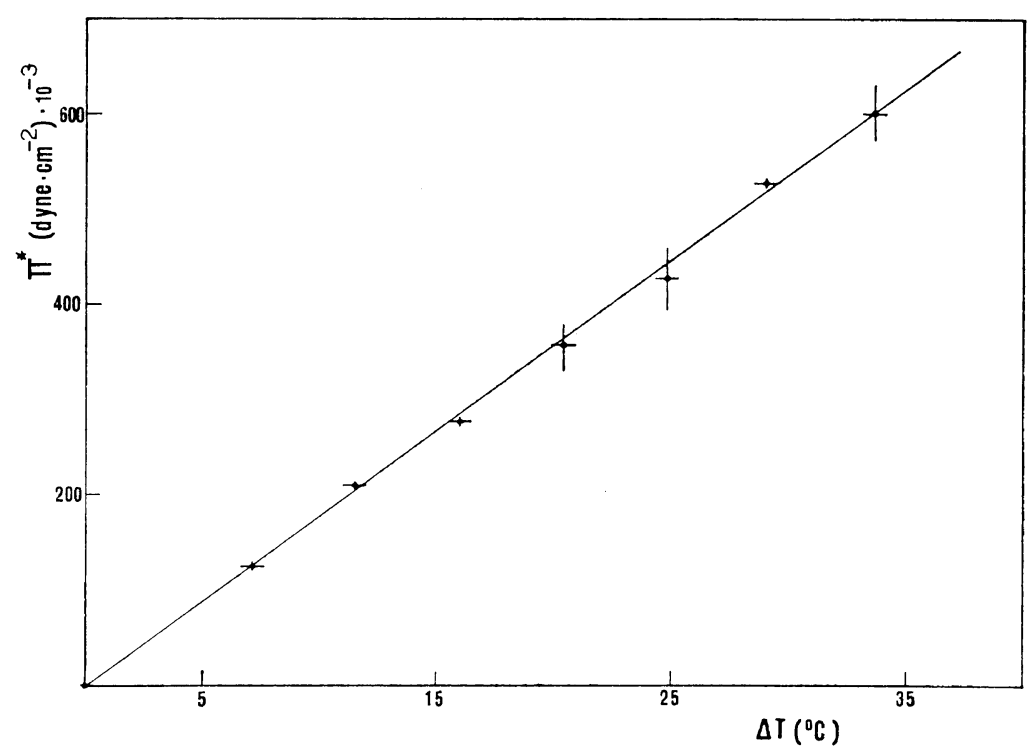

Figure 2. Dependence of the pseudothermoosmotic pressure $\pi^{*}$ upon the temperature difference $\Delta T$ applied to the composite membrane.

mean temperature $T_{\mathrm{m}}=43.4^{\circ} \mathrm{C}$ and $\mathrm{PEO}$ concentration $c=2.0 \%$ inside the chamber LM. By applying a temperature difference $\Delta T$ to the composite membrane, the direction of the flow of matter $J_{\mathrm{M}}$, under the above experimental conditions, was found to be from the cold $R_{2}$ to the hot $R_{1}$ side of the system (see Figure 1) and the pressure reached to the steady state was measured on $R_{1}$. In Figure 2 the dependence on $\Delta T$ of the steady-state pressure, thereafter indicated as "pseudothermoosmotic" pressure $\pi^{*}$, is reported. As it can be seen, the values of $\pi^{*}$, reached by using a composite membrane system, are, as previously verified, far higher than those measured by other authors ${ }^{5-12}$ with simple cellulose membranes. The trend in Figure 2 clearly shows, within the limits of the experimental errors, a linear dependence of the "pseudothermoosmotic" pressure with $\Delta T$. In Figure $3 J_{\mathrm{M}}$ is plotted $v s . \Delta T$, for two different hydrostatic pressures applied to the system.

As it may be seen, a linear dependence of $J_{\mathrm{M}}$ on $\Delta T$, in both the conditions of hydrostatic pressure applied, is shown. This means that it is possible to write for $J_{\mathrm{M}}$ an equation as

$$
J_{\mathrm{M}}=k_{1} \Delta P+k_{2} \Delta T
$$

which is of the same type of eq 1 describing the classical thermoosmosis.

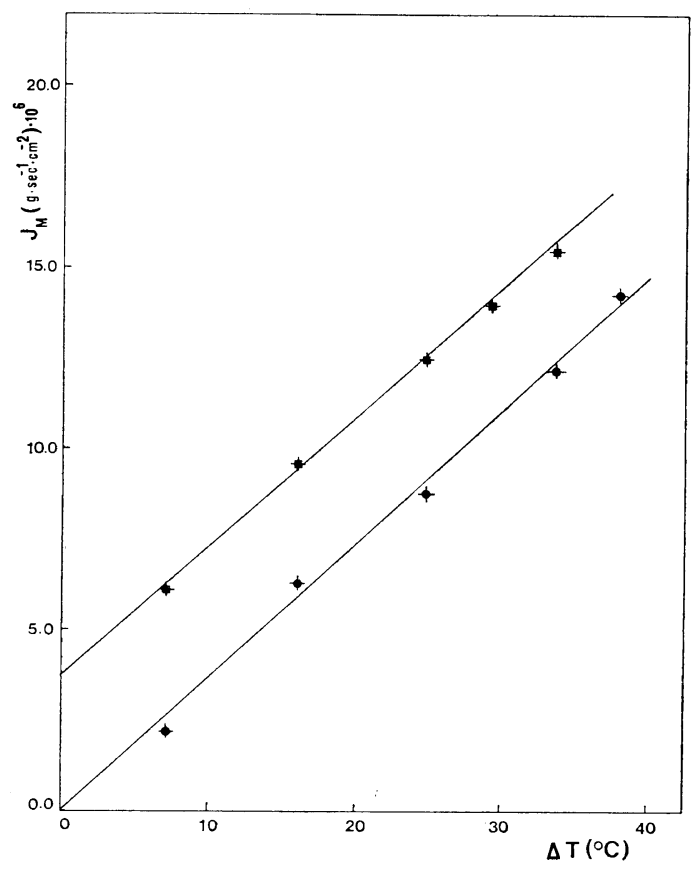

Figure 3. Dependence of the flow of matter $J_{M}$ upon the temperature difference $\Delta T$ applied to the composite membrane: $\bigcirc$, applied hydrostatic pres-

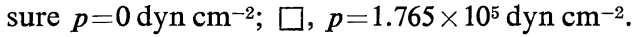




\section{D'Ilario and M. Canella}

It must be emphasized that in our case the flow direction was from the cold to the hot side of the composite membrane which holds for the positive sign of $J_{\mathrm{M}}$, while in usual thermoosmosis experiments $^{5-12}$ it is opposite to that observed by us, at least in the range of the $\Delta T$ applied.

We have then checked the flow direction in the case in which pure solvent was inside LM. The measured flow of matter was $J_{M}=-6.1 \times 10^{-7}$ $\mathrm{g} \mathrm{sec}^{-1} \mathrm{~cm}^{-2}$, roughly in agreement with the Rastogi's ${ }^{12}$ measurements. This evidence strongly supports the hypothesis that, in the case of composite membranes, the effect depends on the physicochemical properties of the solution used as liquid membrane. In order to explain the phenomenon, we have examined two possible models: a, structural gradient liquid membrane model; b, "thermodiffusive" membrane model.

The first one is based on the assumption that some kind of structure arises in the PEO-water system, because of its thermodynamic characteristics. Under the temperature gradient the system should develop a structure gradient; in fact the hydrogen bonds, which are responsible this structural organization, are more stable at then at high temperature. The structural model (micellar, cluster) proposed for the PEOwater system by Elworthy and Florence ${ }^{14}$ should hold in this case.

This hypothesis, however, seems to be insufficient to account for the entity of the measured effect. $^{15}$ In fact the structure effect in the PEOwater solution is not so cooperative as in the case of the conformational transition from helix to coil, under $\mathrm{pH}$ gradient, of the polylysine used in the Liquori's composite membrane. ${ }^{16}$

The second hypothesis is based on a phenomenon typical of closed, nonisothermal systems at one or more components, in a gas or liquid phase, i.e., the thermal diffusion, ${ }^{1-4,17}$ or Soret effect for liquid phases. It consists in the formation of a concentration gradient under the influence of a temperature gradient. As we said above, this effect is peculiar of closed systems, therefore the phenomenon we have observed cannot be explained simply on these bases. As a matter of fact, in our system the two membranes $m_{1}$ and $m_{2}$ allow one of the two components, the solvent, to cross. The assumption we make is that thermal diffusion of the com- ponent which cannot cross $m_{1}$ and $m_{2}$ occurs inside the composite membrane. A similar interpretation to experiments on polystyrene membranes swollen by toluene was given by Voellmy. ${ }^{18}$ Haase, as well, defining a membrane model as "solubility membrane," 19 gives support to this idea.

A possible theoretical model describing our membranes by means of the thermodynamics of irreversible processes will be now discussed. It is worthwhile, at this point, to emphasize that in such a membrane a considerable degree of freedom is present, compared to the thin cellulose membranes used in usual thermoosmosis experiments. In the latter case, in fact, the membrane can be considered as a rigid framework in which the macromolecules are immobilized. In our system the two cellulose membranes $m_{1}$ and $m_{2}$ have only the function of impermeable walls to the PEO macromolecules which, on the contrary, are free to move inside the "liquid membrane." The dissipation function for the composite membrane can be writ$\operatorname{ten}^{4,20}$ as the sum of the two separate dissipation functions at each membrane

$$
\begin{aligned}
\Phi=T \sigma & =T \frac{\mathrm{d}_{i} S}{\mathrm{~d} t}=\phi_{\mathrm{m}_{1}}+\phi_{\mathrm{m}_{2}} \\
& =J_{q}\left(-\frac{\Delta T}{T}\right)+J_{\mathrm{M} 1}\left(\Delta \mu_{\mathrm{m}_{1}}^{1}+\Delta \mu_{\mathrm{m}_{2}}^{1}\right)
\end{aligned}
$$

where $J_{q}$ is the heat flow conjugate to the temperature difference $\Delta T$ across both membranes $\mathrm{m}_{1}$ and $\mathrm{m}_{2}$.

In the condition of stationary flow, the flow of matter $J_{\mathrm{M} 1}$ of component 1, i.e., the solvent, must be the same throughout any part of the system, while the flow of matter $J_{\mathrm{M} 2}$ of component 2, i.e., the macromolecule, is equal to zero, in that the membranes $\mathrm{m}_{1}$ and $\mathrm{m}_{2}$ are impermeable to it. $\Delta \mu_{\mathrm{m}_{1}}^{1}$ and $\Delta \mu_{\mathrm{m}_{2}}^{1}$ are the chemical potential differences relative to component 1 across each membrane $m_{1}$ and $m_{2}$. By making the approximation that $T_{\mathrm{o}_{1}}=T_{\mathrm{i}_{1}}$ and $T_{\mathrm{o}_{2}}=T_{\mathrm{i}_{2}}$, it will be possible to consider just the concentration and pressure-dependent parts of the chemical potential. The second terms of eq 7 can then be rearranged to give

$$
\begin{aligned}
J_{\mathrm{M} 1}\left(\Delta \mu_{\mathrm{m}_{1}}^{1}+\Delta \mu_{\mathrm{m}_{2}}^{1}\right) & =J_{\mathrm{M} 1}\left[\left(\mu_{\mathrm{o}_{1}}^{1}-\mu_{\mathrm{i}_{1}}^{1}\right)+\left(\mu_{\mathrm{i}_{2}}^{1}-\mu_{\mathrm{O}_{2}}^{1}\right)\right] \\
& =J_{\mathrm{M} 1}\left(\Delta \mu_{\mathrm{i}_{21}}^{1}-\Delta \mu_{\mathrm{O}_{21}}^{1}\right)
\end{aligned}
$$


In this expression the chemical potential differences across the "outside" $o_{1}$ and $o_{2}$, and the "inside" $i_{1}$ and $i_{2}$ replace those across $m_{1}$ and $\mathrm{m}_{2}$.

If the entropy production function $\sigma$ is used instead of the dissipation function $\Phi$, a driving force $X_{\mathrm{M} 1}$ conjugate to the flow of component 1 through the composite membrane will be given by

$$
X_{\mathrm{M} 1}=X_{\mathrm{M} 1}^{\mathrm{o}}+X_{\mathrm{M} 1}^{\mathrm{i}}=-\left(\frac{\Delta \mu_{\mathrm{o}_{21}}^{1}}{T_{\mathrm{m}}}\right)+\left(\frac{\Delta \mu_{\mathrm{i}_{21}}^{1}}{T_{\mathrm{m}}}\right)
$$

where $X_{\mathrm{M} 1}^{\mathrm{o}}$ is the driving force for the component 1 acting between outside 1 and outside 2, without knowing anything about the inside of the membrane; $X_{\mathrm{M} 1}^{\mathrm{i}}$ is the driving force for the same component acting inside $L M$, between $i_{1}$ and $\mathrm{i}_{2} . \quad \Delta \mu_{\mathrm{o}_{21}}^{1}$ and $\Delta \mu_{\mathrm{i}_{21}}^{1}$ are the chemical potential differences (concentration and pressure dependent) of the component 1 between the subsystems $o_{2}$ and $o_{1}$, and $i_{2}$ and $i_{1}$, respectively. For $X_{\mathrm{M} 1}^{\mathrm{o}}$ we can write

$$
X_{\mathrm{M} 1}^{\mathrm{o}}=-\left(\frac{\Delta \mu_{\mathrm{o}_{21}}^{1}}{T_{\mathrm{m}}}\right)=-v_{T_{\mathrm{m}}} \frac{\Delta P_{\mathrm{o}_{21}}}{T_{\mathrm{m}}}
$$

and for $X_{M 1}^{\mathrm{i}}$, taking into account the composition of the solution.

$$
X_{\mathrm{M} 1}^{\mathrm{i}}=\left(\frac{\Delta \mu_{\mathrm{i}_{21}}^{1}}{T_{\mathrm{m}}}\right)=v_{T_{\mathrm{m}}}\left(\frac{\Delta P_{\mathrm{i}_{21}}}{T_{\mathrm{m}}}\right)+\frac{1}{T_{\mathrm{m}}}\left(\frac{\partial \mu_{\mathrm{i}}{ }^{1}}{\partial c_{\mathrm{i}}{ }^{1}}\right) \Delta c_{\mathrm{i}}{ }^{1}
$$

An approximation is made by considering the specific volume of the permeant, in all the points of LM, equal to the specific volume at the mean temperature $v_{T_{\mathrm{m}}}$

$$
\begin{aligned}
X_{\mathrm{M} 1} & =-\frac{1}{T_{\mathrm{m}}}\left[v_{T_{\mathrm{m}}}\left(\Delta P_{\mathrm{o}_{21}}-\Delta P_{\mathrm{i}_{21}}\right)-\left(\frac{\partial \mu_{\mathrm{i}}{ }^{1}}{\partial c_{\mathrm{i}}{ }^{1}}\right) \Delta c_{\mathrm{i}}{ }^{1}\right] \\
& =-\frac{1}{T_{\mathrm{m}}}\left[v_{T_{\mathrm{m}}}\left(\Delta P_{\mathrm{o}_{21}}-\Delta P_{\mathrm{i}_{21}}\right)-R T_{\mathrm{m}} \ln \left(\frac{c_{\mathrm{i}_{2}}^{1}}{c_{\mathrm{i}_{1}}^{1}}\right)\right]
\end{aligned}
$$

being $c_{\mathrm{o}_{2}}^{1}=c_{\mathrm{o}_{1}}^{1}$.

The force $X_{q}$ which gives rise to the heat flow is

$$
X_{q}=\frac{1}{T}=-\frac{\Delta T}{T_{\mathrm{m}}{ }^{2}}
$$

The phenomenological equations will be

$$
\begin{aligned}
J_{M 1}= & L_{11} X_{\mathrm{M} 1}+L_{12} X_{q}=-L_{11} \frac{v_{T_{\mathrm{m}}}}{T_{\mathrm{m}}}\left[\left(\Delta P_{\mathrm{o}_{21}}-\Delta P_{\mathrm{i}_{21}}\right)\right. \\
& \left.-\frac{R T_{\mathrm{m}}}{v_{T_{\mathrm{m}}}} \ln \left(\frac{c_{\mathrm{1}_{2}}^{1}}{c_{\mathrm{i}_{1}}^{1}}\right)\right]-L_{12} \frac{1}{T_{\mathrm{m}}{ }^{2}} \Delta T
\end{aligned}
$$

$$
\begin{aligned}
J_{q}= & L_{21} X_{\mathrm{M} 1}+L_{22} X_{q}=-L_{21} \frac{v_{T_{\mathrm{m}}}}{T_{\mathrm{m}}}\left[\left(\Delta P_{\mathrm{o}_{21}}-\Delta P_{\mathrm{i}_{21}}\right)\right. \\
& \left.-\frac{R T_{\mathrm{m}}}{v_{T_{\mathrm{m}}}} \ln \left(\frac{c_{\mathrm{i}_{2}}^{1}}{c_{\mathrm{i}_{1}}^{1}}\right)\right]-L_{22} \frac{1}{T_{\mathrm{m}}{ }^{2}} \Delta T
\end{aligned}
$$

At this point it is necessary to make some considerations. Due to osmotic effects, the composite membrane reaches a mechanical equilibrium, corresponding to the maximum dilution of PEO inside LM, after a certain time. On the assumption that our system, under the influence of $\Delta T$, behaves as a thermal diffusive device for the macromolecule, $\Delta P_{\mathrm{i}_{21}}$ may be neglected and it will be possible to substitute in the eq 12 and 13

$$
\begin{gathered}
\Delta P_{\mathrm{i}_{21}}=0 \\
\ln \frac{c_{\mathrm{i}_{2}}^{1}}{c_{\mathrm{i}_{1}}^{1}}=\frac{\Delta c_{\mathrm{i}_{21}}^{1}}{c_{\mathrm{i}}^{1}}=-K \Delta T
\end{gathered}
$$

$K$ being a constant which relates linearly $\Delta_{c}$ and $\Delta T$, and gives reason for the degree of separation reached by the two components. The phenomenological equations will then assume the following form.

$$
\begin{gathered}
J_{\mathrm{M} 1}=-L_{11} \frac{v_{T_{\mathrm{m}}}}{T_{\mathrm{m}}} \Delta P_{\mathrm{o}_{21}}-\left(L_{12}+L_{11} K R T_{\mathrm{m}}{ }^{2}\right) \frac{\Delta T}{T_{\mathrm{m}}{ }^{2}} \\
J_{q}=-L_{21} \frac{v_{T_{\mathrm{m}}}}{T_{\mathrm{m}}} \Delta P_{\mathrm{o}_{21}}-\left(L_{22}+L_{21} K R T_{\mathrm{m}}{ }^{2}\right) \frac{\Delta T}{T_{\mathrm{m}}{ }^{2}}
\end{gathered}
$$

By replacing with

$$
\begin{aligned}
& L_{12}^{*}=L_{12}+L_{11} K R T_{\mathrm{m}}{ }^{2} \\
& L_{22}^{*}=L_{22}+L_{21} K R T_{\mathrm{m}}{ }^{2}
\end{aligned}
$$

the eq 16 and 17 become

$$
\begin{gathered}
J_{\mathrm{M} 1}=-L_{11} \frac{v_{T_{\mathrm{m}}}}{T_{\mathrm{m}}} \Delta P_{\mathrm{o}_{21}}-L_{12}^{*} \frac{\Delta T}{T_{\mathrm{m}}{ }^{2}} \\
J_{q}=-L_{21} \frac{v_{T_{\mathrm{m}}}}{T_{\mathrm{m}}} \Delta P_{\mathrm{o}_{21}}-L_{22}^{*} \frac{\Delta T}{T_{\mathrm{m}}{ }^{2}}
\end{gathered}
$$

It is worthwhile to point out that these equations contain indeed both the usual effects of thermoosmosis and thermal diffusion as particular cases. In fact, if the system has a single component, $K$ is zero and eq 20 becomes

$$
J_{\mathrm{M} 1}=-L_{11} \frac{v_{T_{\mathrm{m}}}}{T_{\mathrm{m}}} \Delta P_{\mathrm{o}_{21}}-L_{12} \frac{\Delta T}{T_{\mathrm{m}}{ }^{2}}
$$

which is identical to eq 1 . 
L. D'Ilario and M. Canella
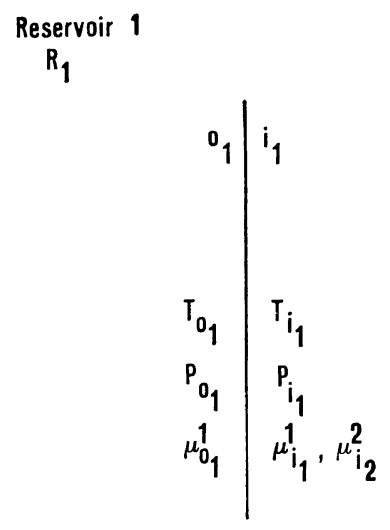

$m_{1}$
Liquid Membrane LM
Reservoir 2

$R_{2}$

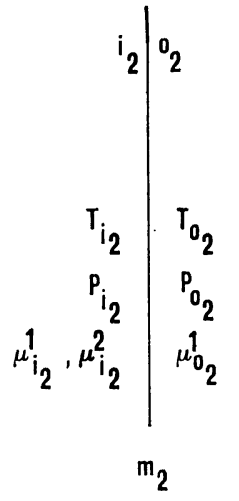

Figure 4. Schematic representation of the system studied: $\mathbf{R}_{1}, \mathbf{R}_{2}$, solvent reservoirs; $\mathrm{m}_{1}, \mathrm{~m}_{2}$, cellulose membranes; $\mathrm{LM}$, liquid membrane; $\mathrm{o}_{1}, \mathrm{o}_{2}$, thin layers of solution on the outside 1 and 2 of the composite membrane; $i_{1}, i_{2}$, thin layers on the inside 1 and 2 of the composite membrane; $T_{\mathrm{o}_{1}}, T_{\mathrm{i}_{1}}, T_{\mathrm{o}_{2}}, T_{\mathrm{i}_{2}}$, temperatures on $\mathrm{o}_{1}, \mathrm{i}_{1}, \mathrm{o}_{2}, \mathrm{i}_{2}, ; \mu_{\mathrm{o}_{1}}^{1}, \mu_{\mathrm{i}_{1}}^{1}, \mu_{\mathrm{o}_{2}}^{1}, \mu_{\mathrm{i}_{2}}^{1}, \mu_{\mathrm{o}_{1}}^{2}, \mu_{\mathrm{i}_{1}}^{2}, \mu_{\mathrm{o}_{2}}^{2}, \mu_{\mathrm{i}_{2}}^{2}$, chemical potentials of components 1 (solvent) and 2 (PEO) on $\mathrm{o}_{1}, \mathrm{i}_{1}, \mathrm{o}_{2}, \mathrm{i}_{2}$.

In the same manner, if $\mathrm{m}_{1}$ and $\mathrm{m}_{2}$ in Figure 4 are completely impermeable to both the components 1 and 2 , and the system is in mechanical equilibrium conditions, eq 20 reduces to

$$
J_{\mathrm{M} 1}=-L_{11} K R \Delta T-L_{12} \frac{\Delta T}{T_{\mathrm{m}}{ }^{2}}
$$

and applying the eq 15

$$
J_{\mathrm{M} 1}=-L_{11} R \frac{\Delta c}{c}-L_{12} \frac{\Delta T}{T_{\mathrm{m}}{ }^{2}}
$$

which is a simplified expression of the phenomenological equation describing the Soret effect.

At the steady state, for $J_{M 1}=0$, eq 20 gives

$$
\left(\frac{\Delta P_{\mathrm{o}_{21}}}{\Delta T}\right)_{J_{\mathrm{M} 1}=0}=-\frac{L_{12}^{*}}{L_{11}} \frac{1}{v_{T_{\mathrm{m}}} T_{\mathrm{m}}}=-\frac{Q^{*}}{v_{T_{\mathrm{m}}} T_{\mathrm{m}}}
$$

where, as for $L_{12} / L_{11}$ in usual thermoosmosis, the ratio $L_{12}^{*} / L_{11}$ has the meaning of a heat of transfer, thereafter indicated by the symbol $\overline{Q^{*}}$

$$
\overline{Q^{*}}=\frac{L_{12}^{*}}{L_{11}}=\frac{L_{12}}{L_{11}}+K R T_{\mathrm{m}}{ }^{2}
$$

Eq 26 can be written as

$$
\overline{Q^{*}}=Q_{\mathrm{TO}}^{*}+Q_{\mathrm{TD}}^{*}
$$

being

$$
Q_{\mathrm{TO}}^{*}=\frac{L_{12}}{L_{11}}
$$

and

$$
Q_{\mathrm{TD}}^{*}=K R T_{\mathrm{m}}^{2}
$$

whereas $Q_{\mathrm{TO}}^{*}$ is the heat of transfer in usual thermoosmosis, $Q_{\mathrm{TD}}^{*}$ represents the heat of transfer for the thermal diffusion effect arising inside the liquid membrane. If the Soret coefficient $S_{\mathrm{T}}$ is substituted to $K$ in eq 26 , the eq 27 becomes

$$
\overline{Q^{*}}=Q_{\mathrm{TO}}^{*}+Q_{\mathrm{SE}}^{*}
$$

where $Q_{\mathrm{SE}}^{*}$ is the heat of transfer for the Soret effect. It is to be noted that in the "pseudothermoosmotic" effect the direction of the flow of matter can be mainly due to the sign of $K$ in eq 16. In our case, using the PEO water solution as liquid membrane, the sign of $K$ must be negative, because of the heat of mixing of PEO in water: the mixing is exothermic. This means that, under the temperature gradient, the PEO will concentrate on the cold side of $\mathrm{LM}$, making $\Delta c_{\mathrm{i}_{21}}^{1}<0$. In order to have a flow of matter from the cold to the hot side of the system i.e., $J_{\mathrm{M} 1}$ positive, the following relation must be verified.

$$
\left|L_{12}\right|<\left|L_{11} K R T_{\mathrm{m}}{ }^{2}\right|
$$

For $K$ ranging between $10^{-5}$ and $10^{-3} \mathrm{deg}^{-1}$, which is the range of the Soret coefficients found in the literature, ${ }^{2}$ taking into account for $L_{12}$ 
Pseudothermoosmosis in a Composite Membrane System

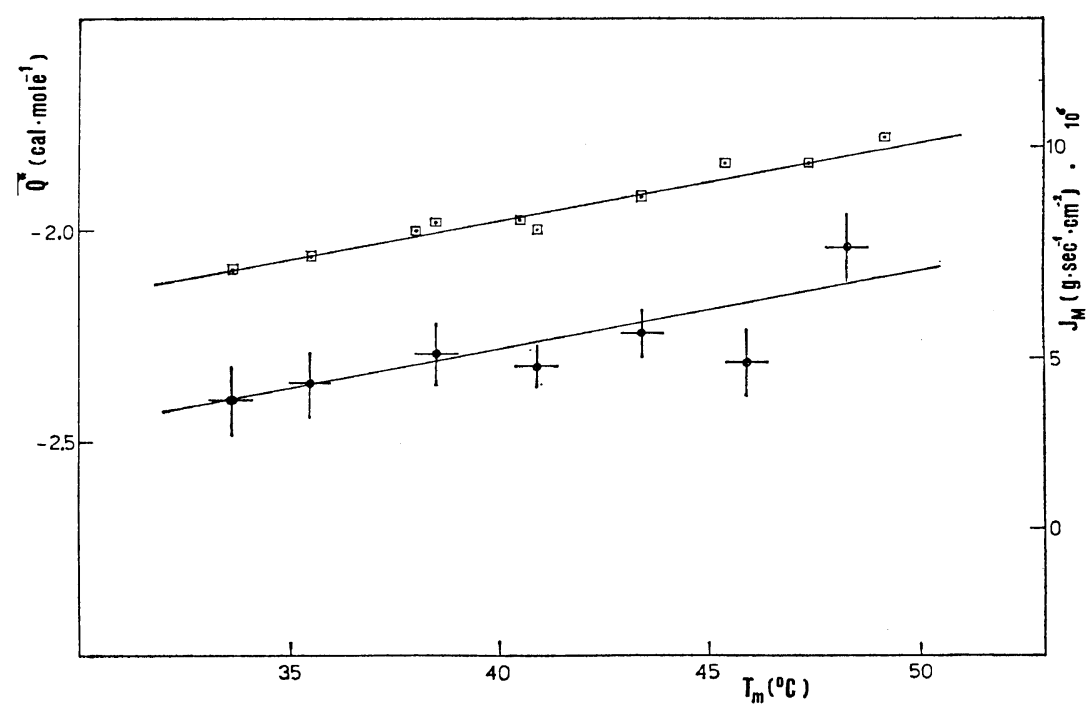

Figure 5. Dependence of $Q^{*}(\bigcirc)$ and of $J_{\mathrm{M}}(\square)$ upon the mean temperature $T_{\mathrm{M}}$.

and $L_{11}$ the Rastogi's value, ${ }^{12}$ eq 31 gives

$$
\begin{aligned}
L_{12} & =4.02 \times 10^{-1} \mathrm{~g} \mathrm{sec}^{-1} \mathrm{~K}<L_{11} S_{\mathrm{T}} R T_{\mathrm{m}}{ }^{2} \\
& =7.1 \div 6.7 \times 10^{2} \mathrm{~g} \mathrm{sec}^{-1} \mathrm{~K}
\end{aligned}
$$

In Figure $5, \overline{Q^{*}}$ is plotted against $T_{\mathrm{m}}$.

In Figure 6 , the trends of $L_{11}$ and $L_{12}^{*}$ are shown vs. $T_{\mathrm{m}}$.

An Arrhenius plot obtained by measuring at different temperatures the flow of matter $J_{\mathrm{M}}$ is

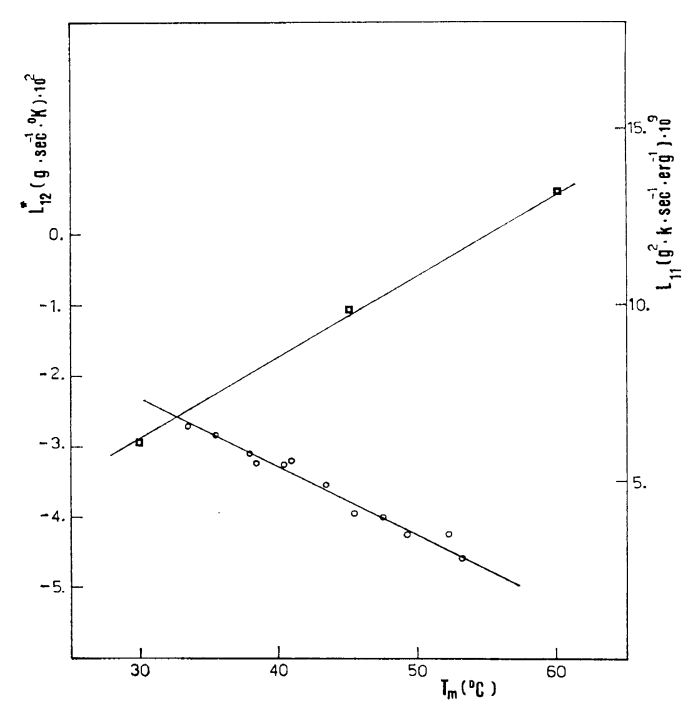

Figure 6. Dependence of $L_{12}^{*}(\bigcirc)$ and of $L_{11}(\square)$ upon the mean temperature $T_{\mathrm{M}}$.

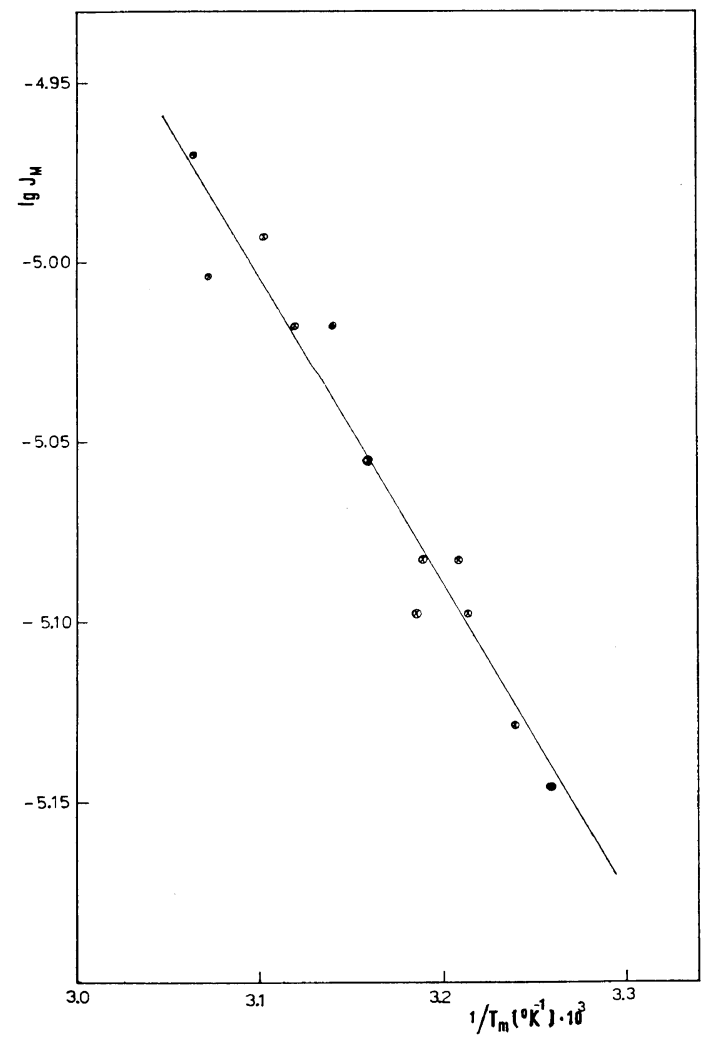

Figure 7. Arrhenius plot of the flow of matter $J_{\mathrm{M}}$ for the solvent transport through the composite membrane. 


\section{D'Ilario and M. Canella}

shown in Figure 7. From it a value of 3.91 $\mathrm{kcal} \mathrm{mol}^{-1}$ is calculated for the apparent activation energy. This is very close to that obtained by Lonsdale, Merten, and Riley ${ }^{21}$ using simple cellulose acetate membranes and applying $\Delta P$ as driving force.

From this value of $\Delta E^{\neq}$they deduce the diffusive nature of the flow of matter through the membrane.

\section{CONCLUSIONS}

As far as we know, this is the first time that a "composite membrane", which we have studied, has been used in thermoosmosis experiments.

As we have already pointed out, the experiment we have presented in this paper cannot be described by means of the phenomenological equations given in the literature for the usual thermoosmosis. ${ }^{5-12}$ In fact, in the "pseudothermoosmotic" effects we have investigated, phenomena as those described of possible formation of structural gradients and of thermal diffusion may play a very critical role. The asymmetry character given to the composite membrane in nonisothermal conditions by all of these phenomena must be considered responsible of the observed effects. They can be described in terms of the irreversible processes thermodynamics by the equations derived in this paper. The main feature in this model is that the equations contain both the usual thermoosmosis and thermal diffusion effects as particular cases, so that they can be used as general equations to describe the transport effects arising in membranes under temperature gradient.

A study of the pseudothermoosmotic pressures and flows dependence on the concentration of PEO inside the liquid membrane is the object of another communication. ${ }^{15}$ Particularly high values of $\pi^{*}$ (ca. $14 \mathrm{~kg} \mathrm{~cm}^{-2}$ ) were observed in those trials. It is our opinion that the amplitude of the measured pseudothermoosmotic pressures is sufficient evidence for the importance of the solute-solvent interactions in membrane systems being far from equilibrium conditions. We think also that this kind of approach could be useful in the description of many biological membrane phenomena which now are explained just in terms of surface interactions.
Acknowledgments. The authors are indebted to Professor A. M. Liquori for having suggested the idea of studying composite membranes in thermoosmosis. The skillful technical assistance of Mr. P. L. Renzi was deeply appreciated.

\section{REFERENCES}

1. S. R. De Groot, "Thermodynamics of Irreversible Processes," North Holland Publ. Co., Amsterdam, 1966.

2. S. R. De Groot and P. Mazur, "Non-equilibrium Thermodynamics," North Holland Publ. Co., Amsterdam, 1969.

3. I. Prigogine, "Thermodynamics of Irreversible Processes," Interscience Publ., New York, N.Y., 1967.

4. A. Katchalsky and P. F. Curran, "Non-equilibrium Thermodynamics in Biophysics," Harvard Univ. Press, Cambridge, Mass., 1967.

5. G. Lippmann, Compt. Rend., 145, 104 (1907).

6. M. Aubert, Ann. Chim. Phys., 26(8), 145 (1912).

7. E. Ernst, Nature, 141, 80 (1938).

E. Ernst and J. Kockzas, Z. Phys., 109, 625 (1938).

E. Ernst, Z. Kolloid, 87, 276 (1939).

8. A. Ursprung, Protoplasma, 33, 200 (1939).

9. N. Riehl, Z. Electrochem., 49, 306 (1943).

10. R. Haase and C. Steinert, Z. Phys. Chem., 21, 270 (1959).

11. C. W. Carr and K. Sollner, J. Electrochem. Soc., 109(7), 616 (1962).

12. R. P. Rastogi, R. L. Blokhra, and R. K. Agarwal, Trans. Faraday Soc., 60, 1386 (1964). R. P. Rastogi and K. M. Jha, J. Phys. Chem., 70, 1017 (1966).

R. P. Rastogi and K. M. Jha, Trans. Faraday Soc., 62, 585 (1966).

R. P. Rastogi and K. Singh, ibid., 62, 1754 (1966).

13. L. D'Ilario and M. Canella, Italy Patent 23557 A/73 (filed 30 April 1973).

14. P. H. Elworthy and A. T. Florence, KolloidZ.Z. Polym., 208(2), 157 (1966).

15. L. D'Ilario and M. Canella, Polymer, 18206 (1977).

16. A. M. Liquori, B. Pispisa, and G. Acampora, La Ricerca Scientifica, 58, 308 (1969).

17. A. Jost, "Diffusion," Academic Press, New York, N.Y., 1970.

18. von H. Voellmy and P. Läuger, Ber. Bunsenges. Phys. Chem., 70(2), 165 (1966).

19. R. Haase, Z. Phys. Chem., 51, 315 (1966).

20. O. Kedem and S. R. Caplan, Trans. Faraday Soc., 61, 1897 (1965).

21. H. K. Lonsdale, U. Merten, and R. L. Riley, J. Appl. Polym. Sci., 9, 1341 (1965). 